\title{
Cluster candidates from the USNO-A2.0 catalogue
}

\author{
A. J. Drake $e^{1,2}$ \\ ${ }^{1}$ Dept. of Astrophysical Sciences, Princeton University, Princeton, NJ 08544, USA \\ e-mail: ajd@astro.princeton.edu \\ 2 Depto. de Astronomia y Astrofisica, P. Universidad Catolica, Vicuña Mackenna 4860, Santiago 22, Chile
}

Received 30 June 2004 / Accepted 28 January 2005

\begin{abstract}
We present the results of a systematic search of the USNO-A2.0 astrometric catalogue for stellar clusters which may have been missed in previous searches performed by eye. This analysis results in the rediscovery of a few hundred known open and globular clusters and the discovery of eight new candidate open clusters.
\end{abstract}

Key words. Galaxy: open clusters and associations: general - galaxies: clusters: general

\section{Introduction}

In recent years there have been a number of studies aimed at finding local star clusters using newly available imaging data and proper motion surveys (Ivanov et al. 2002; Reylé \& Robin 2002; Dutra \& Bica 2000, 2001; Hurt et al. 2000). Many of these surveys have taken advantage of the new near-IR sky surveys DENIS ( $I, J$ and $K$ ) (Cioni et al. 2000) and 2MASS ( $J$, $H$ and $K_{\mathrm{s}}$ ) (Cutri et al. 2003) ${ }^{1}$, as their observations are far less affected by high reddening in the Galactic plane than optical observations. The 2MASS and DENIS images reach stars with $J$ band magnitudes of $\sim 16$.

Given the recent near-IR cluster discoveries we thought it would be interesting to examine optical data using the same methods developed in these new searches. The USNO-A2 all-sky astrometric catalogue contains $B$ and $R$ photographic magnitudes of stars and galaxies down to $V=21$ (Monet 1998). The difference between the limiting magnitude of the USNO-A2 catalogue in $V$ band and the $J$ band limit of the 2MASS point source catalogue is $\sim 5 \mathrm{mag}$. The intrinsic $V-$ $J$ colour of an M5V star is $\sim 5.2$ (Bessell \& Brett 1988), while earlier type dwarfs stars have smaller $V-J$ colours. Therefore, in the absence of dust-induced extinction the USNO-A2 catalogue reaches all dwarf stars earlier than $\mathrm{M} 5 \mathrm{~V}$ to a greater distance than 2MASS. However, in reality there is always reddening along a line-of-sight.

The amount of extinction due to dust in $J$ band is about $25 \%$ that in the $V$ band. For $K$ band the amount of extinction is only $10 \%$ that observed $V$ band (Schlegel et al. 1998). The $V-J$ colour of an M2V star is $\sim 3.2$. Therefore, if we assume that a line-of-sight has a reddening of 2 mag in $V$ (moderately high), then the USNO-A2 catalogue will reach stars earlier than

\footnotetext{
1 www.ipac.caltech.edu/2mass/releases/allsky/ doc/explsup.html
}

M2V type to a greater distance than the 2MASS catalogue. Based on a Salpeter mass function, the total number of stars fainter than M2V type in a young cluster is similar to the number of brighter stars. If clusters are found by their stellar density alone, then both catalogues have similar sensitivity at this reddening level. The USNO catalogue has the depth advantage in searches for clusters in regions where the $V$ band reddening is $\$ 2 \mathrm{mag}$. In areas where the extinction is greater than about 5 mag in $V$, such as regions very near the Galactic plane, the 2MASS observations are deeper.

The average $V$ magnitude extinction of the 720 open clusters from Dias et al. (2002) which have reddening measurements is 1.3 . Only $20 \%$ have a reddening greater than 2 mag. Therefore, one might naively expect to find many clusters using the USNO-A2 catalogue. However, the average measured reddening is biased toward lower values because high extinctions would have made them undetectable at optical wavelengths. For example, Ivanov et al. (2002) found cluster candidates with up to 20 mag of visual extinction, effectively undetectable in optical images. In Fig. 1 we present the locations of the Dias et al. (2002) clusters with measured reddenings. This figure demonstrates the increase in extinction with heliocentric distance and toward the Galactic center. The absence of open clusters greater than a few kilo-parsecs from the Sun clearly shows that many open clusters remain to be found within the Galaxy. Near-IR observations should be able to penetrate much of the dust which has obscured clusters in the less populated regions of this figure. Furthermore, visual observations have been extensively searched by eye for clusters for many years, unlike the near-IR data. For instance, most of the original POSS-I and ESO-R photographic images, on which the USNO-A2 catalogue is based, have been searched by eye (Setteducati \& Weaver 1962; Lauberts 1982; Saurer et al. 1994; Bica \& Schmitt 1995). However, many of the cluster candidates 


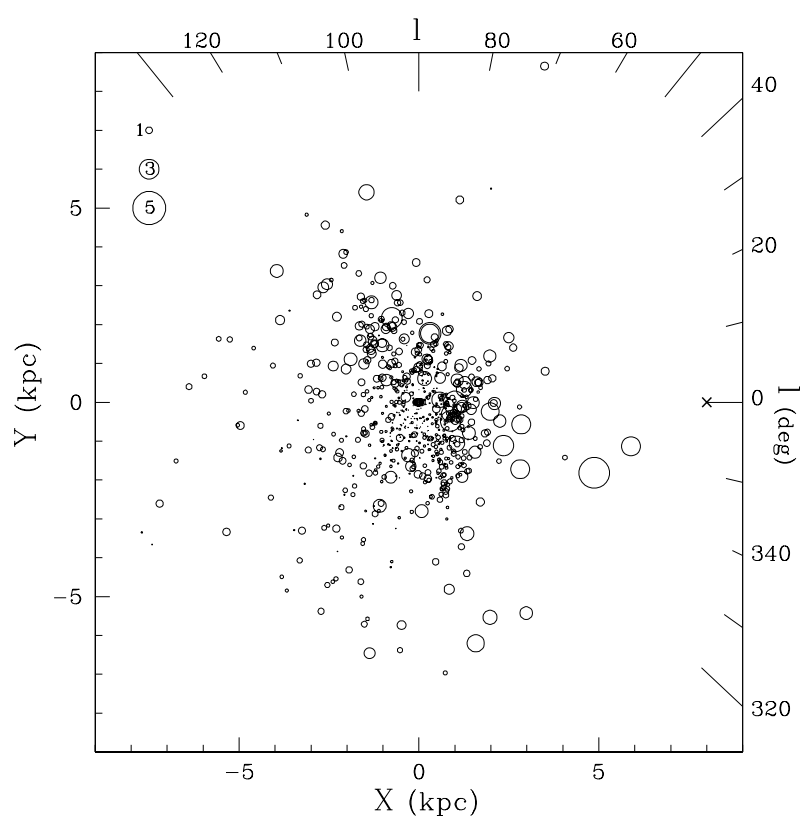

Fig. 1. The distribution of the 720 clusters with known reddenings and distances in the Dias et al. (2002) WEBDA catalogue. Open clusters are marked as circles with sizes proportional to their observed extinction. The galactic center is marked with a cross at a heliocentric distance of $8 \mathrm{kpc}$. Three examples of the sizes of symbols corresponding to 1,3 , and 5 mag of extinction are given in the upper left corner.

discovered in these searches have not been confirmed with photometry or even confirmed by the visual inspections by others (Dias et al. 2002). Cluster searches based primarily on statistics rather than visual perception should provide improved consistency.

A recent search for open clusters has also been carried out by Alessi et al. (2003) using the Tycho-2 astrometric catalogue (Hog et al. 2000). In this search the proper motions and CMDs of the stars were used to find 11 new open cluster candidates. The Tycho- 2 catalogue consists of 2.5 million stars to $V_{\mathrm{T}} \sim 11.5$. In contrast the USNO-A2 astrometric catalogue does not contain proper motions but has half a billion objects with a limit of $V=21$. At the limiting magnitude of the Tycho- 2 catalogue, the proper motions of the individual stars within a cluster are useful in determining membership. However, at fainter magnitudes the stars are more likely to be too distant to provide accurate proper motions (on average). Furthermore, the proper motions of faint stars are less certain than bright stars because their centroid locations have larger errors. Therefore, proper motion information is unlikely to be accurate enough to use in searches for the faintest clusters.

The recently released USNO-B1 astrometric catalogue (Monet et al. 2003) is also well suited for a stellar cluster search. This catalogue contains twice the number of objects as the USNO-A2 and includes additional photometric information as well as proper motion and star-galaxy separation data. However, the full catalogue has not been distributed, and although it is possible to search the USNO-B1 catalogue on$\operatorname{line}^{2}$, it is currently not in a form practical to download.

\footnotetext{
${ }^{2}$ http://www.nofs.navy.mil/data/FchPix/
}

\section{Cluster search}

In order to discover stellar clusters that may have been missed in previous searches we follow the simple method of Ivanov et al. (2002) and Reylé \& Robin (2002). Firstly, we converted the USNO equatorial coordinates to Galactic coordinates since this is a more appropriate system for a cluster search. Next, we performed an Aitoff-projection of the coordinates. This projection gives us regions of equal area on the sky. We then divided the transformed sky coordinates into bins and determined the number of stars in each region and look for bins with unexpectedly large numbers of stars.

In order to be sensitive to globular clusters and open clusters we binned the USNO-A2 catalogue stars using two different box scales, $1.5^{\prime}$ and $2.5^{\prime}$. Our choice of box size was based on Ivanov et al. (2002). These authors found that the average half-mass radius for globular clusters in the Harris (1996) catalogue is $1.3 \pm 0.8^{\prime}$ and the average size of Galactic open clusters in the Lyngå (1995) catalogue is $3.5 \pm 2.5^{\prime}$. For the open cluster search we used a smaller box size than the average as we expected that undiscovered clusters would likely be more distant and therefore smaller. In contrast, globular clusters are not concentrated locally and thus are unlikely to be much smaller than the average value. Nearby clusters larger than 5 arcmin are likely to be missed because of the small box size. It is also more difficult to detect such large clusters because the amount of background contamination is higher. As the detection of a cluster within any box is dependent on where the highest concentration of stars lies relative to the edge of the bin, we binned all the stars in two passes (at each bin scale) using a half bin offset in each coordinate.

The cluster candidate selection of Ivanov et al. (2002) required that the number of stars in a bin was $3 \sigma$ greater than the background and that there were more than 50 stars in the bin. In our search we did not use a uniform $\sigma$ cut above the background level and the number of stars required for selection was also varied. We discovered this was necessary since the fluctuation in the number of background stars is highly variable near the Galactic disk because of the patchiness of extinction. However, in most cases we required at least a $4 \sigma$ deviation from the background number of stars and more than 12 stars in any bin. In all cases we determined the number of background stars by averaging the number of stars in the 16 bins which were offset by 2 bins from the bin being tested. This offset between the candidate bin and the background determination region lowers the possibility that stars from the cluster will increase the measured background level.

During our initial tests we found many cluster candidates with many times the background number of stars. Most of these high signal-to-noise detections were discovered near the locations of very bright stars. Upon examination of the DSS-2 images it was clear that these were due to reflection and diffraction artifacts in the images. To reduce the number of false detections we matched our candidate positions with the locations of stars in the SAO and Tycho-2 catalogues (Hog et al. 2000) and removed candidates within $1.25^{\prime}$ of known bright stars. After removing the artifacts from our candidates, our list contained a few thousand locations of candidate clusters. For each of the 
candidates we extracted a $7^{\prime} \times 7^{\prime}$ Red DSS-2 image from the ESO On-line Digitized Sky Survey. This image database is useful since it currently covers $98 \%$ of the sky. Candidates falling in the remaining $2 \%$ region were examined using DSS-2 images obtained from the Simbad database ${ }^{3}$.

Although the inspection of the candidates by eye is undesirable due to the subjective nature of this process we found it to be unavoidable at this point. The selection was aided by comparison of the candidates with well known clusters within these same DSS-2 images. Nevertheless, in many cases it was difficult to discern whether a perceived cluster was real or simply an artifact. We chose to remove all the candidates where there was some degree of doubt. In regions where the stellar density was high it was particular difficult to identify clusters by eye because of the presence of unrelated bright stars. It was also difficult to detect clusters in these regions purely statistically because of the high background and the fluctuations in the background due to extinction variations. These factors are likely to have led to a bias toward lower stellar density regions in our analysis.

The locations of the candidate clusters were matched with known clusters within $5^{\prime}$ using the NED and SIMBAD online astronomical databases and the $\mathrm{WEBDA}^{4}$ open cluster database of Dias et al. (2002). We found it was necessary to use this large matching radius because it was clear that many of our best candidates belonged to known clusters whose coordinates were offset by a few arcminutes in these databases.

\section{Results and discussion}

In Fig. 2 we present images of each candidate stellar cluster extracted from the DSS-2. Among our candidates we also include ESO cluster 370-SC017 which is marked as not-found in the Dias et al. (2002)'s WEBDA catalogue. In our analysis we also discovered a few clusters of objects far from the galactic plane. In most cases the existence of extended objects makes it clear that these are clusters of galaxies. In Fig. 3 we present images of the galaxy clusters discovered.

In order to check the validity of each stellar cluster candidate we extracted the data from the USNO-B1 catalogue which has twice as many objects as the USNO-A2 catalogue. We used this data to determine the projected stellar density as a function of distance from the cluster center. For each case the cluster center was determine as the location of the peak of the stellar density. In Fig. 4 we present density profiles of the stellar cluster candidates with King (1962) model profile fits to each. Clearly the small number of stars in these clusters leds to a high degree of uncertainty in the King model fits. The density profiles of the galaxy cluster candidates are not shown as the USNO-B1 catalogue does not accurately represent the total number of galaxies present. We adopt labels DC and DGC for stellar and galaxy cluster candidates, respectively. In Table 1 we present the parameters of the cluster candidates. We note that all the candidates lie in the region $80^{\circ} \leq l \leq 300^{\circ}$. Most were found in directions of low extinction, as seen in Fig. 1.

\footnotetext{
${ }^{3}$ http://simbad.u-strasbg.fr/Simbad

${ }^{4}$ http://obswww. unige.ch/webda/
}

In addition, the amount of confusion due to high stellar crowding is low in these fields.

For greater certainty about the nature of the candidate open clusters it is necessary to photometry of the associated stars. Unfortunately the USNO-B1 catalogue photometry is of poor quality with a standard deviation of $\sim 0.25 \mathrm{mag}$ (Monet et al. 2003). The photometric accuracy is hindered by the nonlinear response of the photographic plates. 2MASS sky survey near-IR data is available for all of these clusters. However, in most cases this photometry is too shallow to be of any use. As the available photometric data is of poor quality we have not attempted to determine the ages of the clusters through isochrone fitting of the available data. Follow-up observations of some of the candidates have been carried out and is the subject of a separate analysis (Drake 2005).

In our search we did not find any new Galactic globular cluster candidates. There are 147 globular clusters in the Harris (1996) catalogue yet we found only 44 of these. Such clusters should be relatively easy to find due to their high stellar density relative to the the background. Upon examination we discovered that the main region for our poor detection efficiency was because the USNO-A2 catalogue does not cover the central regions of the globular clusters. This is likely to be due to the core of the clusters being saturated in the photographic images used to produce the source catalogue. The USNO-B1 catalogue does cover the cluster cores but is clearly incomplete. However, we believe that it would be useful to search the USNO-B1 catalogue for new globular clusters. We note that globular clusters have also recently been discovered in regions inaccessible to optical surveys using near-IR data (Borisova et al. 2003).

As regards open cluster detection, 935 of the 1688 clusters from the Dias et al. (2002) WEBDA catalogue were discovered in our analysis with a significance $3.5 \sigma$ above the background with more than 12 stars per bin, while 504 clusters exceeded a $4 \sigma$ level. Nevertheless, 448 of the 1688 clusters in the WEBDA catalogue are flagged as not found, dubious, possible OB associations, non-existent, dust holes, etc. In addition, 494 of the WEBDA open clusters have radii greater that $5^{\prime}$. As we noted earlier, we have a lower detection sensitivity to large clusters because of our $2.5^{\prime}$ box size. We detected only 140 of the 330 WEBDA catalogue open clusters larger than $5^{\prime}$ that were not flagged. However, in our our selection we found that a $4 \sigma$ or higher significance was necessary as $0.5 \%$ of the bins ( 200000$)$ were selected at the $3.5 \sigma$ level. Among the 504 clusters exceeding $4 \sigma \sim 300$ passed our selection criteria and were viewed by eye and of these, $75 \%$ were discovered in the images during inspection. In light of the large number of clusters flagged in the WEBDA, we are satisfied with our cluster detection sensitivity.

\subsection{Notes on individual candidates}

DC1 is perhaps the poorest candidate cluster with few points above the background level of stars as can be seen in the radial density plot. However, the detection level is higher than many of the other cluster candidates. DC2, 3 and 4 are clear in both the radial density plots and the DSS-2 images of the regions. 

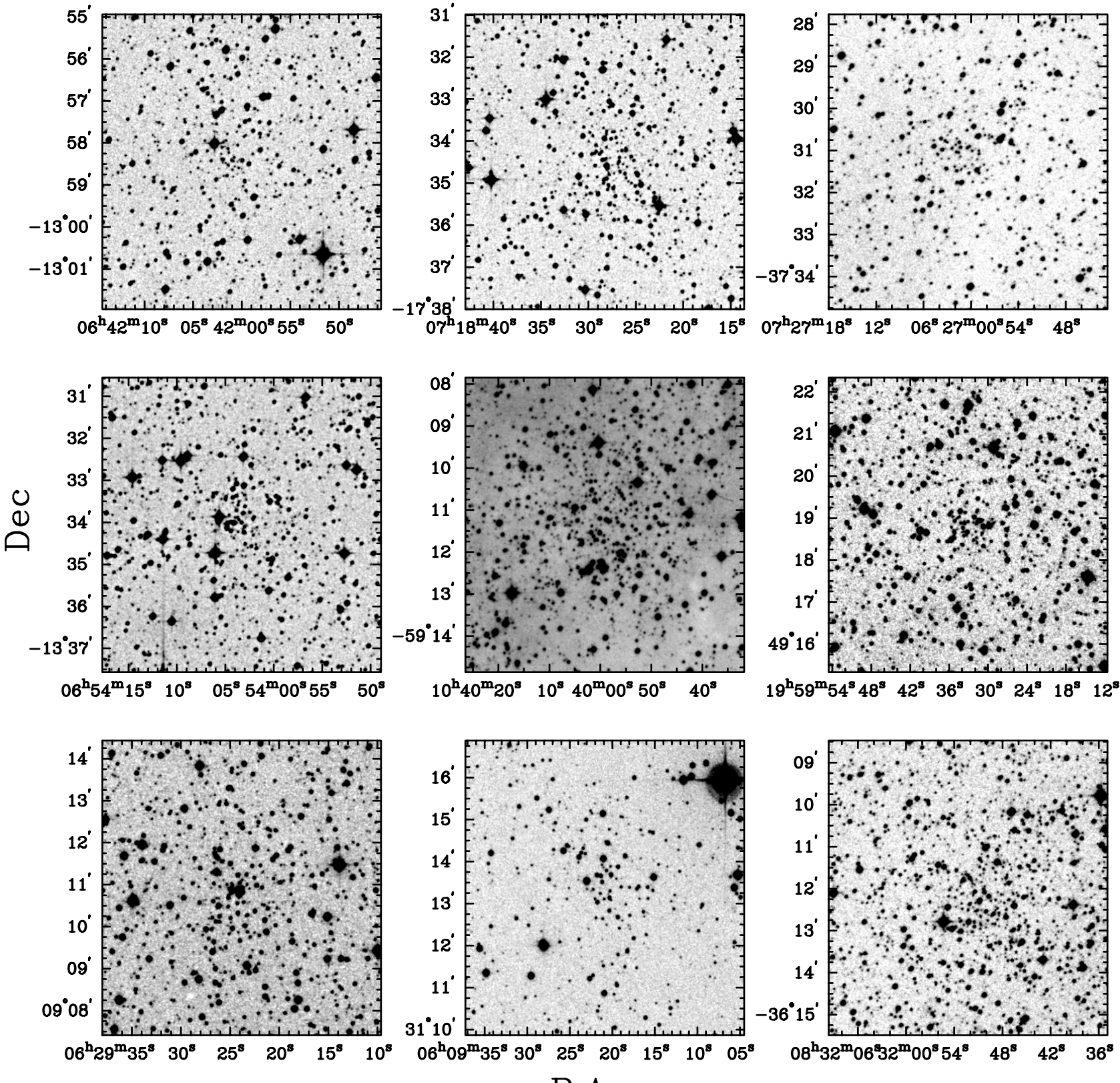

R.A.

Fig. 2. $7^{\prime} \times 7^{\prime}$ DSS-2 $R$-band finding charts for the stellar cluster candidates DC1, DC2, DC3, DC4, DC5, DC6, DC7, DC8 and ESO $370-$ SC 017 in order left to right, top to bottom.

DC5 is very near to the galactic plane and shows the presence of a large amount of differential reddening. From the Schlegel et al. (1998) reddening maps we found $E(B-V) \sim 3.4$. However, Bonifacio et al. (2000) found the values of Schlegel et al. (1998) to be too large in regions of high extinction. We follow Bonifacio et al. (2000)'s correction and obtained $E(B-V) \sim 2.3$. To check that the appearance of this candidate was not an artifact caused by differential reddening we downloaded the 2MASS near-IR $J, H$ and $K_{\mathrm{s}}$ images of the region. These images show an over density of stars and the density profile of sources matches that of the USNO-B1 data. Therefore, we consider this is a good candidate.

DC6, 7 and 8 all show over-densities in the DSS-2 images. Candidate DC7 is very near the Galactic plane like DC5.
However, the image does not show signs of differential reddening effects and this is born out by the relatively low value of $E(B-V)=0.45$.

Cluster ESO 370-017 is a known star cluster (Lauberts 1982). However, the Dias et al. (2002) WEBDA catalogue lists this as not-found (nf) on inspection of DSS images. The cluster appears quite clear in the $R$ band DDS-2 image and the radial density of USNO-B1 objects shows a good signal. The cluster is listed as having an apparent diameter of $3^{\prime}$ which is in agreement with our King model radius of $1.4^{\prime}$.

DGC1, 2, and 3 all appear to be reasonable galaxy cluster candidates. However, DGC3 has a low signal to noise ratio and may simply be the random projection of a few unassociated galaxies. All these candidates are more than $25^{\circ}$ from the 

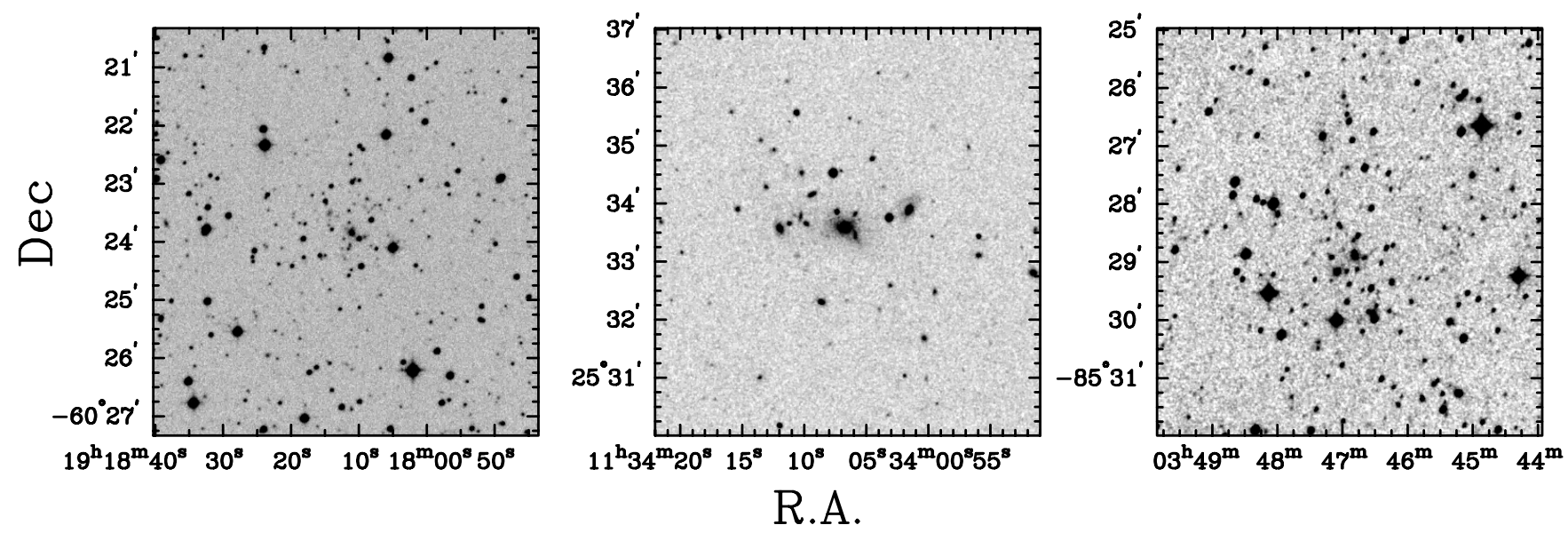

Fig. 3. $7^{\prime} \times 7^{\prime}$ DSS-2 finding charts for galaxy cluster candidates DGC1, DGC2, DGC3, in order left to right.

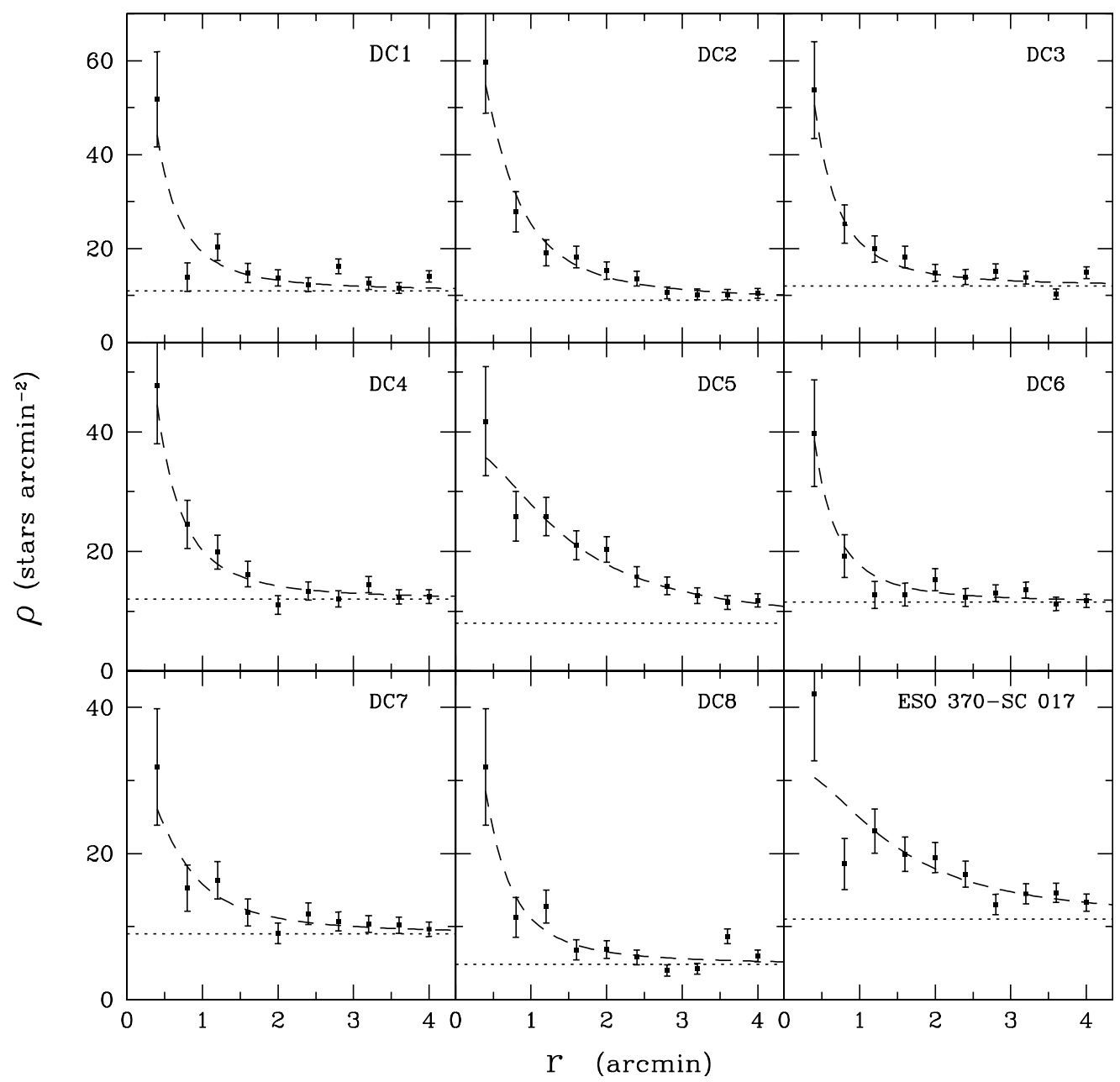

Fig. 4. Radial density profiles for cluster candidates. The dashed lines show the King model for each, while the dotted line shows the approximate stellar background level.

Galactic plane where extinction is low. There is little likelihood that any of these candidates are stellar clusters because of their distance from the Galactic plane and the clear presence of extended objects. Each of these cluster candidate contains galaxies associated with it that were identified by 2 MASS. Redshifts or photometry of moderate quality could easily confirm the nature of these candidates.

\section{Summary}

We have rediscovered a few hundred Galactic open and globular clusters in an automated search of the USNO-A2 catalogue. However, we find only a small number of new galaxy and open cluster candidates and no new Galactic globular cluster candidates. Our search for globular clusters was hampered by the 
Table 1. Cluster candidates. Column 1, ID. Columns 2 and 3, Right Ascension and Declination (J2000). Columns 4 and 5, Galactic Latitude and Longitude. Column 6, Number of stars in detection box. Column 7, Number of standard deviations above background. Column 8, King model core radius.

\begin{tabular}{lccccccc}
\hline \hline ID & $\begin{array}{l}\text { RA } \\
\text { h.m.s }\end{array}$ & $\begin{array}{c}\text { Dec } \\
\circ, "\end{array}$ & $\begin{array}{c}l \\
\circ\end{array}$ & \multicolumn{1}{c}{$b$} & $N$ & $N_{\sigma}$ & $r_{\mathrm{c}}$ \\
& & & & & & \\
\hline DC1 & $06: 42: 00.8$ & $-12: 58: 27$ & 223.49 & -7.93 & 31 & 5.3 & 0.34 \\
DC2 & $07: 18: 28.2$ & $-17: 34: 30$ & 231.60 & -2.17 & 34 & 4.8 & 0.55 \\
DC3 & $07: 27: 00.3$ & $-37: 31: 16$ & 250.28 & -9.70 & 41 & 4.3 & 0.33 \\
DC4 & $06: 54: 03.3$ & $-13: 34: 03$ & 225.34 & -5.57 & 37 & 5.0 & 0.35 \\
DC5 & $10: 39: 59.0$ & $-59: 11: 21$ & 286.79 & -0.50 & 44 & 4.5 & 1.4 \\
DC6 & $19: 59: 33.0$ & $+49: 18: 50$ & 83.64 & 10.13 & 35 & 6.0 & 0.3 \\
DC7 & $06: 29: 23.8$ & $+09: 10: 55$ & 202.26 & -0.67 & 31 & 7.1 & 0.63 \\
DC8 & $06: 09: 20.8$ & $+31: 13: 22$ & 180.58 & 5.59 & 16 & 4.6 & 0.38 \\
ESO & $08: 31: 52.2$ & $-36: 11: 59$ & 255.95 & 2.02 & 47 & 4.4 & $1.4^{a}$ \\
DGC1 & $19: 18: 11.8$ & $-60: 23: 49$ & 336.21 & -26.60 & 24 & 5.7 & $\ldots b^{b}$ \\
DGC2 & $11: 34: 06.5$ & $+25: 33: 31$ & 213.73 & 72.62 & 13 & 13.8 & $\ldots b^{b}$ \\
DGC3 & $03: 46: 53.4$ & $-85: 28: 29$ & 299.31 & -30.34 & 16 & 4.4 & $\ldots b^{b}$ \\
\hline
\end{tabular}

a Cluster ESO 370-SC 017 is already known. However, it is marked as not-found by Dias (2002) from their inspection of DSS images, so we have included it.

${ }^{b}$ Galaxy cluster candidate.

fact that there are objects missing from USNO-A2 catalogue near globular cluster cores. For open clusters, the fact that we find hundreds of known clusters but only a small number of new candidates, leds us to believe that previous cluster searches have been relatively complete.

Acknowledgements. This research has made use of the SIMBAD database operated at CDS, Strasbourg, France. This research has made use of the NASA/IPAC Infrared Science Archive and NASA/IPAC
Extragalactic Database (NED), which are operated by the Jet Propulsion Laboratory, California Institute of Technology, under contract with the National Aeronautics and Space Administration. This work was supported by Chilean FONDECYT grant 1030955.

\section{References}

Alessi, B. S., Moitinho, A., \& Dias, W. S. 2003, A\&A, 410, 565 Bessell, M., \& Brett, J. M. 1988, PASP, 100, 1134

Bica, E. L. D., \& Schmitt, H. R. 1995, ApJS, 101, 41

Borisova, J., Pessev, P., Ivanov, V. D., et al. 2003, A\&A, 411, 83

Bonifacio, P., Monai, S., \& Beers, T. C. 2000, AJ, 120, 2065

Cioni, M. R., Loup, C., Habing, H. J., et al. 2000, A\&AS, 144, 235

Cutri, R. M., Sktrutskie, M. F., Van Dyk, S., et al. 2003, VizieR On-line Data Catalog: II/246 (Originally published in: University of Massachusetts and Infrared Processing and Analysis Center (IPAC/California Institute of Technology))

Dias, W. S., Alessi, B. S., Moitinho, A., \& Lépine, J. R. 2002, A\&A, 389,871

Drake, A. J. 2005, A\&A, submitted

Dutra, C. M., \& Bica, E. 2000, A\&A, 359, L9

Dutra, C. M., \& Bica, E. 2001, A\&A, 376, 434

Harris, W. 1996, AJ, 112, 1487

Hog, E., Fabricius, C., Makarov, V. V., et al. 2000, A\&A, 355, 27

Hurt, R. L., Jarrett, T. H., Kirkpatrick, D., \& Cutri, R. M. 2000, AJ, 120,1876

Ivanov, V. D., Borissova, J., Pessev, P., Ivanov, G. R., \& Kurtev, R. 2002, A\&A, 394, L1

King, I. 1962, AJ, 67, 471

Lauberts, A. 1982, The ESO/Uppsala Survey of the ESO(B) Atlas (Garching: ESO)

Lyngå, G. 1995, VizieR On-line Data Catalog: VII/92A (Originally published in: Lund Observatory)

Monet, D. 1998, BAAS, 30, 1427

Monet, D., Levine, S., Canzian, B., et al. 2003, AJ, 125, 984

Reylé, C., \& Robin, A. C. 2002, A\&A, 384, 403

Saurer, W., Seeberger, R., Weinberger, R., \& Zeiner, R. 1994, AJ, 107, 2101

Schlegel, D., Finkbeiner, D., \& Davis, M. 1998, ApJ, 500, 553

Setteducati, A. F., \& Weaver, M. F. 1962, Newly found stellar clusters, University of California, Berkeley 